\title{
Cohen-Gibson syndrome in a family: The first familial case report
}

\author{
Yeo Jin Kang ${ }^{1 \oplus}$ and Young Ok Kim ${ }^{1,2, * \odot}$ \\ 'Department of Pediatrics, Chonnam National University Childrens' Hospital, Gwangju, Korea \\ ${ }^{2}$ Department of Pediatrics, Chonnam National University Medical School, Gwangju, Korea
}

\begin{abstract}
Cohen-Gibson syndrome (CGS) was first reported by Cohen et al., who identified the mutation of the gene encoding the embryonic ectoderm development (EED) in a patient with phenotypes similar to Weaver syndrome. CGS manifests as an overgrowth and intellectual disability, in addition to the characteristic facial features and organ anomalies. CGS has been reported in only 11 unrelated patients since 2015. A girl aged 6 years and 3 months presented with seizures. She had macrosomia, a dysmorphic face, and intellectual disability. Her mother and younger sister and brother also had macrosomia, intellectual disability, and similar facial features; additionally, her mother experienced seizures and had an arachnoid cyst, while her siblings had valvar pulmonary stenosis. Whole-exome sequencing for the proband revealed a mutation of $E E D$ (c.581A $>\mathrm{G}$, p.Asn194Ser), which was also verified in the mother and both siblings using Sanger sequencing. This is the first report of familial CGS.
\end{abstract}

Key words: Intellectual disability, Growth, Development, Embryonic ectoderm development protein, Seizures.

\section{Introduction}

Overgrowth syndrome can be divided into three categories: (1) overgrowth and intellectual disability (OGID; e.g., Sotos syndrome and Weaver syndrome); (2) overgrowth without intellectual disability (e.g., Beckwith Wiedemann syndrome and Marfan syndrome); (3) asymmetric or regional overgrowth (e.g., Proteus syndrome and Klippel-Trenaunay-Weber syndrome) $[1,2]$. The genetic causes of OGID have been increasingly elucidated, given the rapid development of next-generation sequencing $[1,2]$. In 2017, Tatton-Brown et al. [2] identified pathogenic genetic mutations in 353 of 710 (50\%) patients with OGID through targeted gene analyses and exome sequencing. Fourteen genes, including the gene encoding the embryonic ectoderm development (EED), were found in the OGID group [2].

The heterozygous mutation of EED was first identified in 2015 by Cohen et al. [3] in a patient presenting with phenotypes resembling Weaver syndrome, and it was subsequently named Cohen-Gibson syndrome (CGS). Only nine variants of EED (NM_003797.4, NP003788.2) have been reported in 11 unrelated patients [2-10]; however, there has been no familial case. Patients with CGS demonstrate macrosomia, developmental delay, and intellectual disability [2-10]. The characteristic craniofacial features of CGS are evolving $[3-5,7,8]$. The typical facial phenotypes of CGS are identifiable during infancy and early childhood $[3-5,7,8]$. The abnormalities in patients with CGS are generally skeletal, ocular, auditory, neurologic, cardiac, genitourinary, gastrointestinal, dermatologic, and dental in nature (Table 1) [2-10].

Received: 23 December 2020, Revised: 29 January 2021, Accepted: 29 January 2021, Published: 30 June 2021

*Corresponding author: Young Ok Kim, M.D., Ph.D. (iD https://orcid.org/0000-0002-7873-1140

Department of Pediatrics, Chonnam National University Childrens' Hospital, 42 Jebong-ro, Dong-gu, Gwangju 61469, Korea.

Tel: +82-62-220-6646, Fax: +82-62-222-6103, E-mail: ik052@jnu.ac.kr

Conflict of interest: The authors declare that they do not have any conflicts of interest.

(ㄷ) This is an open-access article distributed under the terms of the Creative Commons Attribution Non-Commercial License (http://creativecommons.org/licenses/by-nc/4.0/) which permits unrestricted non-commercial use, distribution, and reproduction in any medium, provided the original work is properly cited.

(c) Copyright 2021 by the Korean Society of Medical Genetics and Genomics 
Table 1. Clinical characteristics of total 15 patients with EED mutations

\begin{tabular}{|c|c|}
\hline Somatic phenotypes & $\begin{array}{l}\text { Overgrowth (100\%), macrocephaly (60.0\%), large slender feet (53.3\%), large hands and fingers (46.6\%), wide } \\
\text { nipples (13.3\%) }\end{array}$ \\
\hline Facial features & 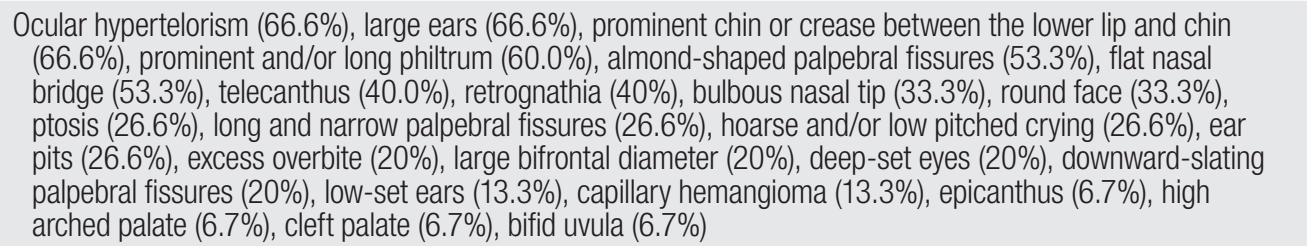 \\
\hline $\begin{array}{l}\text { Developmental and } \\
\text { neurologic features }\end{array}$ & $\begin{array}{l}\text { Intellectual disability and developmental delay (100\%), unsteady gait (33.3\%), abnormal finding on brain MRI } \\
(26.6 \%) \text {, epilepsy (20\%), provoked seizure due to hyperinsulinemic hypoglycemia }(6.7 \%)\end{array}$ \\
\hline Ocular and auditory features & $\begin{array}{l}\text { Myopia (26.6\%), strabismus (20\%), hearing loss (13.3\%), cataract (6.7\%), hyperopic astigmatism (6.7\%), large } \\
\text { lacrimal gland (6.7\%) }\end{array}$ \\
\hline Musculoskeletal issues & $\begin{array}{l}\text { Advanced bone age }(40 \%) \text {, hypertonia }(40 \%) \text {, kyphoscoliosis }(40 \%) \text {, broad metaphyses }(33.3 \%) \text {, hypotonia } \\
(26.6 \%) \text {, vertebral fusion or stenosis }(26.6 \%) \text {, camptodactyly }(26.6 \%) \text {, joint hypermobility }(26.6 \%) \text {, osteope- } \\
\text { nia }(13.3 \%) \text {, Erlenmeyer flask deformity }(6.7 \%) \text {, hip dysplasia }(6.7 \%) \text {, coxa valga }(6.7 \%)\end{array}$ \\
\hline $\begin{array}{l}\text { Gastrointestinal and } \\
\text { genitourinary features }\end{array}$ & $\begin{array}{l}\text { Umbilical hernia (40\%), cryptorchidism (20\%), hydronephrosis (13.3\%), duplicated collecting system }(6.7 \%) \text {, } \\
\text { Hirschsprung disease and intestinal malrotation }(6.7 \%) \text {, inguinal and femoral hernia }(6.7 \%) \text {, bowel obstruction } \\
(6.7 \%) \text {, cholecystitis }(6.7 \%)\end{array}$ \\
\hline Others & $\begin{array}{l}\text { Heart anomalies (53.3\%), abnormal fingernails }(33.3 \%) \text {, several pigmented nevi }(26.6 \%) \text {, hyperinsulinemic } \\
\text { hypoglycemia (13.3\%), dysplastic enamel (13.3\%), adrenal insufficiency with adrenal gland calcification }(6.7 \%) \text {, } \\
\text { bruxism }(6.7 \%) \text {, tracheomalacia }(6.7 \%) \text {, lipodystrophy }(6.7 \%) \text {, thin/fragile skin }(6.7 \%) \text {, soft doughy skin }(6.7 \%)\end{array}$ \\
\hline \multicolumn{2}{|c|}{$\begin{array}{l}\text { Total } 15 \text { patients exhibited } 9 \text { different EED mutations as follows: c.581A>G (p.Asn194Ser) in } 6 \text { patients; c.772C>T (p.His258Tyr) in } 2 \text { patients; and } 1 \\
\text { patient for each of the following mutations, c.706A>G (p.Arg236Gly); c.707G>C (p.Arg236Thr); c.710A>G (p. Asp237Gly); c.733A>T (p.His258Leu); } \\
\text { c.904A>G (p.Arg302Gly); c.906A>C (p.Arg302Ser); c.917_919delinsCGG (p.Arg306_Asn307delinsThrAsp). } \\
\text { Data from the article of Tatton-Brown K et al. (Am J Hum Genet 2017;100:725-36) [2]; Cohen AS et al. (J Hum Genet 2015;60:339-42) [3]; Cohen AS et al. (J } \\
\text { Hum Genet 2016;61:831-4) [4]; Cooney E et al. (Am J Med Genet A 2017;173:541-5) [5]; Imagawa E et al. (Hum Mutat 2017;38:637-48) [6]; Smigiel R } \\
\text { et al. (J Hum Genet 2018;63:517-20) [7]; Cyrus S et al. (Am J Med Genet C Semin Med Genet 2019;181:519-31) [8]; Griffiths S et al. (Am J Med Genet A } \\
\text { 2019;179:588-94) [9]; Spellicy CJ et al. (J Hum Genet 2019;64:561-72) [10]. }\end{array}$} \\
\hline
\end{tabular}

In this study, we attempted to determine the genetic etiologies of epilepsy and familial OGID using whole-exome sequencing (WES) in a female patient admitted at our hospital. The heterozygous missense mutation of EED (c.581A $>\mathrm{G}$, p.Asn194Ser), which has been reported in two CGS patients $[2,9,10]$, was found. However, to the best of our knowledge, this is the first case report of familial CGS with EED mutation.

This study was approved by the Human Research Ethics Committee of Chonnam National University Hospital (IRB number CNUH-2017-167). Informed consent was obtained from her parents. The biospecimens were provided by the Chonnam National University Hospital Biomedical Research Institute Biobank according to the protocols approved by the review board.

\section{Case}

A girl aged 6 years and 3 months presented with status epilepticus with generalized tonic-clonic seizures followed by a right hemi-convulsion. At 3 years of age, she suffered a complex febrile convulsion (a focal non-motor seizure followed by bilateral tonic-clonic seizures). She was $132 \mathrm{~cm}$ in height (>97 percentile) and $35.1 \mathrm{~kg}$ in weight (>97 percentile). Her head circumference was $53 \mathrm{~cm}$ (90-95 percentile). She had a round and flat face, almond-shaped palpebral fissures, ocular hypertelorism, telecanthus, a low nasal bridge, a prominent long philtrum, a mildly prominent chin with a horizontal chin crease, large hands, and large slender feet. However, she had no joint hypermobility. She was delivered through a Cesarean section at 38 weeks of gestational age, weighing $4.6 \mathrm{~kg}$ (large for gestational age, $>97$ percentile) after an uneventful pregnancy. The patient previously exhibited developmental delay and moderate intellectual disability. The total intelligence quotient (I0), assessed using the fourth edition of the Korean Wechsler Preschool and Primary Scale of Intelligence, was 48 at 5 years and 5 months of age.

The patient's mother and two siblings (younger sister and brother) had macrosomia, developmental delay, mild-tomoderate intellectual disability, and similar facial features (Fig. 1). The total IQ of the proband's mother was 47 at 35 years of age; it was tested using the fourth edition of Korean Wechsler Adult Intelligence Scale. The total IOs of the proband's younger sister and brother were 64 (at 5 years of age) and 67 (at 4 years and 11 months of age), respectively. The mother experienced seizures at around 30 years of age and had been on antiepileptic drugs. Additionally, the mother had a large arachnoid cyst in the left 


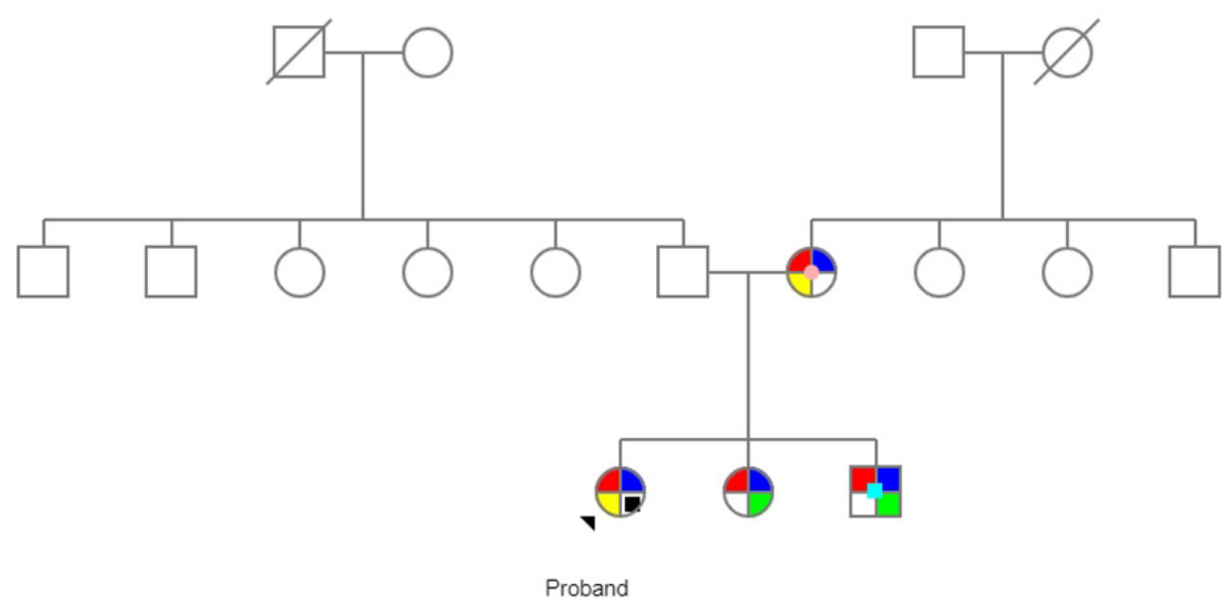

Fig. 1. Family pedigree. The four family members with overgrowth, intellectual disability, and dysmorphic facial features had epilepsy (in the proband and mother), cardiac anomalies (valvar pulmonary stenosis with or without a persistent left superior vena cava in both siblings), a large arachnoid cyst (in the mother), and genitourinary anomalies (hydronephrosis and cryptorchidism in the younger brother). They were diagnosed with Cohen-Gibson syndrome based on whole-exome sequencing and/or Sanger sequencing. middle and anterior cranial fossae with a mass effect on the left frontal and temporal lobes. It was incidentally detected by brain computed tomography and magnetic resonance imaging (MRI). The proband's younger sister had valvar pulmonary stenosis. The proband's younger brother also had valvar pulmonary stenosis and a persistent left superior vena cava. He had cryptorchidism of the left testis and bilateral mild hydronephrosis that spontaneously improved a year after birth.

Serum electrolytes, blood glucose, and blood lactate levels in the proband were all within normal limits. Electroencephalography showed no epileptiform discharges. Brain MRI showed no abnormalities, although it demonstrated symmetric enlargement of the lacrimal glands. A radiograph of the skeletal systems showed neither abnormalities nor advanced bone age. Echocardiography and abdominal ultrasound showed no abnormalities. The proband had no hearing disability. Ophthalmologic examination showed intermittent exotropia. Chromosomal analysis, chromosomal microarray study, and blood amino acid analysis showed no abnormal results.

WES was performed for the proband using the SureSelect Human All Exon V5 (Agilent Technologies Inc., Santa Clara, CA, USA) on the NextSeq 500 System (IIlumina Inc., San Diego, CA, USA). Variant calling was performed using the Genome Analysis Toolkit, version 3.5, Best Practices (Broad Institute, Cambridge, MA, USA). The Korean Reference Genome Database (http://coda. nih.go.kr/coda/KRGDB/index.jsp) was selected as one of the ref-
Father

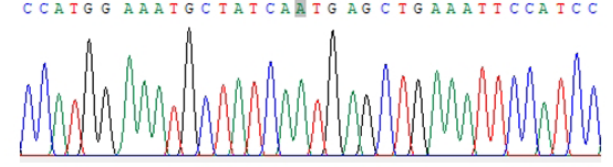

Proband

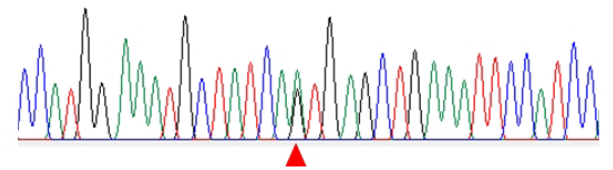

Younger sister

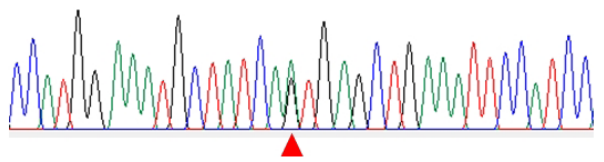

Younger brother

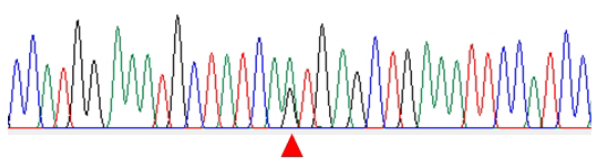

Mother

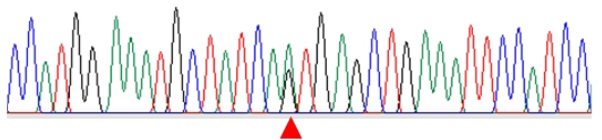

Fig. 2. Electropherogram. The electropherogram showed the likely pathogenic heterozygous missense mutation (c.581A >G, p.Asn194Ser) of EED (NM_003797.4) in the proband, both siblings, and the mother and the wild-type sequence in the father.

erence databases for single-nucleotide polymorphisms. The proband's WES indicated a likely pathogenic heterozygous missense mutation (c.581A>G, p.Asn194Ser) of EED (NM_003797.4). 
The variant was verified in the proband, the mother, and both siblings through Sanger sequencing; a wild-type sequence was identified in the father (Fig. 2).

The proband was on topiramate for seizure control. When the seizures recurred one and a half years later, topiramate was replaced with oxcarbazepine. The proband has been seizure-free for approximately 4 years. The younger sister and brother experienced no seizures until their last visit at 8 years and 7 months of age and 5 years and 3 months of age, respectively. Both siblings had no skeletal abnormalities during their last visit.

\section{Discussion}

To our knowledge, only 15 cases of CGS, including the four current familial patients in this report, have been reported [210]. This makes it very rare. Based on the data from 15 patients with CGS (Table 1), CGS is characterized by a tall stature or macrosomia from the prenatal period (100\%), slightly variable facial dysmorphism (100\%), macrocephaly (60\%), large hands and feet (53.3\%), and global developmental delay with intellectual disability (100\%) to a variable degree (from mild to severe) [210]. Dysmorphic facial features include hypertelorism, telecanthus, almond-shaped palpebral fissures, large ears, a flat nasal bridge, a prominent and long philtrum, and a prominent chin with a crease [2-10]. The characteristic musculoskeletal features include advanced bone age, kyphoscoliosis, and flared metaphyses [2-10]. Other anomalies include heart anomalies (53.3\%), mildly abnormal brain MRI findings (26.6\%), myopia/strabismus, genitourinary anomalies, and hyperinsulinemic hypoglycemia (13.3\%) [2-10]. Most reported abnormalities in patients with CGS are not critical [2-10]. There are no known pathognomonic signs of CGS (Table 1) [2-10]. In contrast with other CGS patients, the six patients with the c.581A>G mutation of EED were less likely to have musculoskeletal phenotypes: none of the current four related patients had anomalies of the musculoskeletal system $[2,9,10]$.

For our familial cases, the heart anomalies of the two siblings were valvar pulmonary stenosis with or without a persistent left superior vena cava, which has not been previously reported for patients with CGS. Previously reported heart anomalies associated with CGS include mitral valve regurgitation, patent ductus arteriosus, atrial septal defect, and ventricular septal defect $[2,3,5,6,9,10]$. The arachnoid cyst discovered in the proband's mother was the first coincidence reported for CGS. Previous abnormal brain MRI findings included substantial white matter volume loss, corpus callosum thinning, enlarged ventricles, a cyst of the septum pellucidum, and a foreshortened corpus callosum $[5,7,10]$. Large lacrimal glands were observed in the proband.

The unprovoked seizures in the proband and her mother were diagnosed as drug-responsive epilepsy. Epilepsy was previously reported in the first patient diagnosed with CGS due to an EED c.906A > C (p.Arg302Ser) mutation [3]. Seizures in this patient were successfully treated with phenytoin and primidone, with reoccurrence only 1-2 times per year [3]. Provoked seizures due to hyperinsulinemic hypoglycemia were reported in one patient with the c.581A $>G$ (p.Asn 194Ser) mutation [2,9]. Clinicians should be aware of the possibility of hyperinsulinemic hypoglycemia in patients with CGS presenting with seizures.

$E E D$, in association with the enhancer of Zeste homolog 2 (EZH2) and the suppressor of Zeste 12 (SUZ12), is a core component in the polycomb repressive complex 2 (PRC2) [2-6,8-11]. As PRC2 demonstrates histone methyltransferase activity, EED, $E Z H 2$, and SUZ12 are considered epigenetic growth regulators [2-6,8-11]. The PRC2-related syndromes caused by the mutations of the abovementioned genes have similar clinical phenotypes, which may be due to their shared pathomechanisms [2,8-10]. However, there are some differences between the syndromes [8-10]. Patients with CGS caused by EED mutations, when compared with patients with Weaver syndrome caused by $E Z H 2$ mutations, show more frequent and severe intellectual disabilities $[8,9]$. Cryptorchidism, umbilical hernias, musculoskeletal abnormalities, and congenital heart disease are more frequent in patients with CGS than in those with Weaver syndrome $[8,9]$.

The EED is located on chromosome $11 q 14.2$ and has a total of 12 coding exons [10]. Among the 9 EED variants reported, c. $581 \mathrm{~A}>\mathrm{G}$ (p.Asn 194Ser) accounts for $40 \%$ of the total 15 patients affected with CGS (Table 1) [2-10]. The c.772C>T (p.His258Tyr) variant was reported in two patients with CGS $[4,10]$. Among the $9 E E D$ variants, eight were heterozygous missense mutations between exon 6 and 9 , whereas only one was indel [2-10]. There is no known genotype and phenotype correlation. Imagawa et al. [6] investigated the functional effects of the atypical protein produced due to the EED mutation (c.707G $>$ C, p.Arg236Thr) and reported the suppression of the PRC2 enzyme activity in vitro.

This is the first report of familial CGS caused by a heterozygous EED mutation demonstrating an autosomal dominant maternal inheritance. Pulmonary stenosis with or without a persistent left superior vena cava, large lacrimal glands, and a large arachnoid cyst were newly described in this family with CGS. 


\section{Acknowledgements}

This research was supported by the Basic Science Research Program through the National Research Foundation of Korea (NRF) funded by the Ministry of Education, Korea (grant no. NRF-2017R1D1A3A03000532).

\section{Authors' Contributions}

Conception and design: YOK. Acquisition of data:YOK, YJK. Analysis and interpretation of data: YOK, YJK. Drafting the article: YOK, YJK. Critical revision of the article:YOK. Final approval of the version to be published: YOK, YJK.

\section{References}

1. Edmondson AC, Kalish JM. Overgrowth syndromes. J Pediatr Genet 2015;4:136-43.

2. Tatton-Brown K, Loveday C, Yost S, Clarke M, Ramsay E, Zacha-

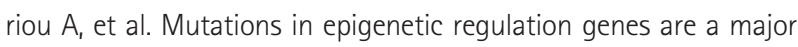
cause of overgrowth with intellectual disability. Am J Hum Genet 2017:100:725-36.

3. Cohen AS, Tuysuz B, Shen Y, Bhalla SK, Jones SJ, Gibson WT. A novel mutation in EED associated with overgrowth. J Hum Genet 2015;60:339-42.

4. Cohen AS, Gibson WT. EED-associated overgrowth in a second male patient. J Hum Genet 2016;61:831-4.
5. Cooney $\mathrm{E}, \mathrm{Bi}$ W, Schlesinger $A E$, Vinson $\mathrm{S}$, Potocki L. Novel EED mutation in patient with Weaver syndrome. Am J Med Genet A 2017;173:541-5.

6. Imagawa E, Higashimoto K, Sakai Y, Numakura C, Okamoto N, Matsunaga $S$, et al. Mutations in genes encoding polycomb repressive complex 2 subunits cause Weaver syndrome. Hum Mutat 2017;38:63748.

7. Smigiel $R$, Biernacka $A$, Biela $M$, Murcia-Pienkowski $V$, Szmida $E_{1}$ Gasperowicz $P$, et al. Novel de novo mutation affecting two adjacent aminoacids in the EED gene in a patient with Weaver syndrome. J Hum Genet 2018;63:517-20.

8. Cyrus S, Burkardt D, Weaver DD, Gibson WT. PRC2-complex related dysfunction in overgrowth syndromes: a review of EZH2, EED, and SUZ12 and their syndromic phenotypes. Am J Med Genet C Semin Med Genet 2019;181:519-31.

9. Griffiths S, Loveday C, Zachariou A, Behan LA, Chandler K, Cole T, et al. EED and EZH2 constitutive variants: a study to expand the CohenGibson syndrome phenotype and contrast it with Weaver syndrome. Am J Med Genet A 2019;179:588-94.

10. Spellicy CJ, Peng Y, Olewiler L, Cathey SS, Rogers RC, Bartholomew D, et al. Three additional patients with EED-associated overgrowth: potential mutation hotspots identified? J Hum Genet 2019;64:561-72.

11. Choufani S, Gibson WT, Turinsky AL, Chung BHY, Wang T, Garg K, et al. DNA methylation signature for $\mathrm{EZH} 2$ functionally classifies sequence variants in three PRC2 complex genes. Am J Hum Genet 2020;106:596-610. 